\section{Pericentric inversion of chromosome 11 in one of two similar retarded brothers}

We report two brothers, aged $2 \frac{1}{2}$ and $4 \frac{1}{2}$, with similar features, namely developmental delay, short stature, and small delicate facies. Chromosome analysis revealed a pericentric inversion of chromosome 11 in one boy and a normal karyotype in the other. The father was shown to be the carrier of this pericentric inversion.

Two brothers with mental retardation, particularly in the area of speech development, were assessed for aetiological factors. Examination revealed that both were short, the younger boy being below the 3rd centile and the older one below the 10th centile for height, while the parents, grandparents, and two older normal sibs were on the 75th centile for height. In both, the facies were small and delicate with prominent bossed forehead. There were no other congenital abnormalities. In both cases pregnancy and delivery were normal, as were their birthweights. There was no apparent exposure to terato-

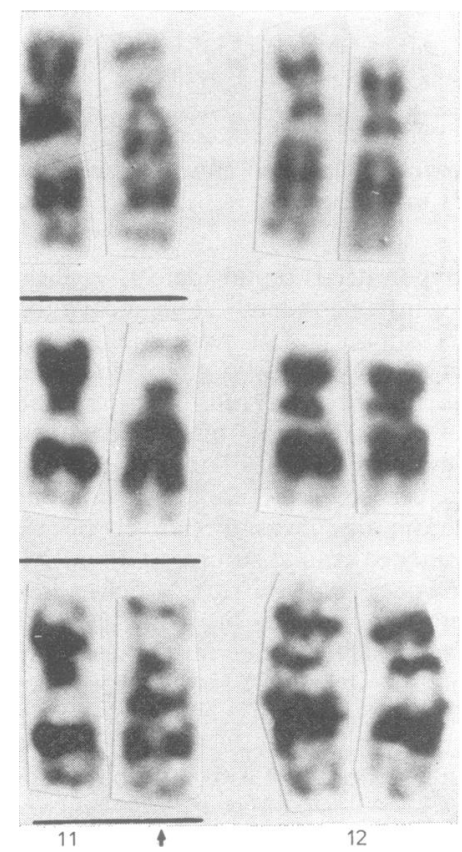

FIGURE Partial $G$ banded karyotype from the proband and his father. The arrow indicates the abnormal chromosome 11.

Received for publication 16 November 1981 genic agents. The boys have not suffered any significant injury or ill-health and there is no evidence of emotional disturbance or psychosocial deprivation. The older boy is functioning in the moderately retarded range and the younger boy in the mildly retarded range. There has been no evidence of regression in attainments. There is no family history of mental retardation. Both have normal hearing.

Chromosome analysis with $\mathrm{G}$ and $\mathrm{C}$ banding revealed a pericentric inversion of chromosome 11 in the younger boy and the father: 46,XY,inv(11)(pl5q14) (figure). Karyotypes of the mother, the older retarded boy, and the two normal sibs were normal. Examination of 50 cells with deprived medium ${ }^{1}$ did not reveal a fragile $X(q 27)$ in either boy. Urinary metabolic screening was normal.

Inversion of chromosome 11 is a rare finding, having been reported in six other cases known to us to date. ${ }^{2}$ All reported cases were balanced inversions and no phenotypic effect was ascribed to the inversion carrier. Our case further illustrates this point, as the inversion was present in only one of the boys and in his normal father. It could not be the cause of the boys' retardation. This chromosome abnormality would not have been detected without the use of banding techniques as it was a structural rearrangement within the $\mathrm{C}$ group. ${ }^{3}$ It may be that inversion of chromosome 11 is more common than suggested by published reports.

The two boys described here had few congenital abnormalities, but their phenotype was similar and they were clearly different to the other family members. Assuming that the mental retardation of these boys has a similar aetiology, it could be the result of unknown environmental factors, sex linked mental retardation, or an autosomal recessive condition.

\section{STEWART L EINFELD AND Arabella SMITH Grosvenor Diagnostic and Assessment Centre, Summer Hill, NSW, and Cytogenetics Unit, Oliver Latham Laboratory,} Health Commission of New South Wales, Australia

\section{References}

1 Sutherland GR. Fragile sites on human chromosomes. Demonstration of their dependence on the type of tissue culture medium. Science 1977;197:265-6.

2 Autio-Harmainen $\mathrm{H}$, de la Chapelle A. High resolution of a small pericentric inversion of chromosome 11 . $J$ Med Genet 1980;17:44-7.

3 Fisher NL, Starr ED, Greene T, Hoehn H. Utility and limitations of chromosome banding in pre- and postnatal service cytogenetics. Am J Med Genet 1980;5:285-94.

Requests for reprints to Dr A Smith, Cytogenetics Unit, Oliver Latham Laboratory, Health Commission of NSW, PO Box 53, North Ryde 2113, Australia. 